\title{
Observations of Multiple Surges Associated with Magnetic Activities in AR10484 on 25 October 2003
}

\author{
Wahab Uddin ${ }^{1}$, B. Schmieder ${ }^{2}$, R. Chandra ${ }^{3}$, Abhishek K. Srivastava ${ }^{1}$, Pankaj Kumar ${ }^{4}$, S. \\ Bisht $^{3}$ \\ wahab@aries.res.in
}

\begin{abstract}
We present a multiwavelength study of recurrent surges observed in $\mathrm{H} \alpha$, UV (SOHO/EIT) and Radio (Learmonth, Australia) from the super-active region NOAA 10484 on 25 October, 2003. Several bright structures visible in $\mathrm{H} \alpha$ and UV corresponding to subflares are also observed at the base of each surge. Type III bursts are triggered and RHESSI X-ray sources are evident with surge activity. The major surge consists of the bunches of ejective paths forming a fan-shape region with an angular size of $\left(\approx 65^{\circ}\right)$ during its maximum phase. The ejection speed reaches upto $\sim 200 \mathrm{~km} / \mathrm{s}$. The SOHO/MDI magnetograms reveal that a large dipole emerges east side of the active region on 18-20 October 2003, a few days before the surges. On October 25, 2003, the major sunspots were surrounded by "moat regions" with moving magnetic features (MMFs). Parasitic fragmented positive polarities were pushed by the ambient dispersion motion of the MMFs and annihilated with negative polarities at the borders of the moat region of the following spot to produce flares and surges. A topology analysis of the global Sun using PFSS shows that the fan structures visible in the EIT $171 \AA$ images follow magnetic field lines connecting the present AR to a preceding AR in the South East. Radio observations of type III bursts indicate that they are coincident with the surges, suggesting that magnetic reconnection is the driver mechanism. The magnetic energy released by reconnection is transformed into plasma heating and provides the kinetic energy for the ejections. A lack of a radio signature in the high corona suggests that the surges are confined to follow the closed field lines in the fans. We conclude that these cool surges may have some local heating effects
\end{abstract}

\footnotetext{
${ }^{1}$ Aryabhatta Research Institute of Observational Sciences (ARIES), Nainital, India.

${ }^{2}$ LESIA, Observatoire de Paris-Meudon, 92195, Meudon Cedex, France.

${ }^{3}$ Department of Physics, DSB Campus, Kumaun University, Nainital, India, 263002.

${ }^{4}$ Korea Astronomy and Space Science Institute (KASI), Daejeon, 305-348, Republic of Korea.
} 
in the closed loops, but probably play a minor role in global coronal heating and the surge material does not escape to the solar wind.

Subject headings: Solar flare - surges, magnetic field, sunspots, magnetic reconnection

\section{INTRODUCTION}

Solar surge is a collimated ejection of plasma material from the lower solar atmosphere into the corona. These ejecta exhibit episodic heating and cooling also, therefore, may be visible in the range of emissions from $\mathrm{H} \alpha$ to EUV/UV, and X-rays, and can be abbreviated in general as solar jets (Schmieder et al. 1995). The surges may have initiation velocity of $\sim 50 \mathrm{~km} \mathrm{~s}^{-1}$, which may further increase up to a maximum value of $100-300 \mathrm{~km} \mathrm{~s}^{-1}$, and these surges may reach up to the heights of $10 \mathrm{Mm}$ to $200 \mathrm{Mm}$ or even more Sterling 2000). Lifetime of surges is about $30 \mathrm{~min}$, and they can be recurrent with a period of an hour or more (Schmieder et al. 1984, 1995). Usually the surge is confined to one or several narrow threads of magnetic fields embedded in the plasma that shoot out above the solar surface. However, such surges are mostly associated with the flaring regions and the sites of solar transients where recurrent magnetic reconnection is dominant. The evolution of solar surges has been studied comprehensively in association with magnetic field emergence and cancellation, as well as flaring activities of the solar atmosphere where such plasma jets also appeared twisted and spiraled (e.g., Schmieder et al. 1994; Chae et al. 1999; Y Yoshimura et al. 2003; Liu \& Kurokawa 2004, and references cited there in).

The solar surges may occur in the regions of emerging magnetic fluxes in the vicinity of satellite spots (Rust 1968; Rov 1973; Kurokawa \& Kawai 1993). Often these surges are associated with flares (Schmieder et al. 1988, 1995; Uddin et al. 2004; Chandra et al. 2006), and magnetic reconnection may be responsible for the acceleration as well as heating of the plasma. An other kind of reconnection could occur due to the collision of opposite polarity magnetic fluxes in "moat region" (Brooks et al. 2007). Small moving magnetic features called MMFs (Harvey \& Harvey 1973; Sainz Dalda \& López Ariste 2007; Kitiashvili et al. 2010), are observed as moat regions. The formation of MMFs is closely related to the fragmentation and disintegration of sunspot magnetic fluxes. Recent studies show that the amount of magnetic flux lost by the sunspots is similar to the flux transported in the moat region (Kubo et al. 2008). The flux is annihilated at the border of the moat region, and in its consequence the subflares, jets and surges may occur during the reconnection processes along neutral lines (Beck et al. 2007; Brooks et al. 2007, 2008; Engell et al. 2011). Theoretical models have been developed concerning canceling flux producing surges or jets. 
The emerging-flux model of Yokovama \& Shibata (1996) supports this kind of surge dynamics, which may be triggered due to the interaction of emerging photospheric field with the pre-existing overlying coronal magnetic fields. Although magnetic reconnection and photospheric magnetic activities may be the key in driving many solar surges and other jets, however, several other mechanisms may also be responsible for the surge/jet dynamics. Pariat et al. (2010) have developed a 3D reconnection model without evidence of emerging flux. This model is able to generate untwisting jets when a stress is constantly applied at the photosphere (Rachmeler et al. 2010). Shibata et al. (1982) and Sterling et al. (1993) have reported that the pressure pulse can trigger the solar surges of moderate heights in the hydrodynamic regime of the solar atmosphere. Solar surges may also be accelerated due to the whip motion of the reconnection generated newly formed magnetic field (Shibata et al. 1992; Canfield et al. 1996a), while the reconnection generated explosive events may also trigger such kind of plasma dynamics (Madjarska et al. 2009). In addition to the typical solar surges, Georgakilas et al. (1999) have observed the polar surges as cool jets at polar region without any association with transients. Recently, the cool jets and surges have also been modeled respectively in the polar region as well as near the boundary of a non-flaring active region due to reconnection generated velocity pulses in the ideal magnetohydrodynamic (MHD) regime of the solar atmosphere (Srivastava \& Murawski 2011; Kayshap et al. 2012). In conclusions, the solar surges and other various types of solar jets may be excited via both, e.g., the direct magnetic reconnection processes in the emerging field regions, as well as due to the magnetohydrodynamic wave activities.

During October-November 2003, major solar activity originated from three super-active regions, namely NOAA AR 10484, 10486 and 10488. The active region NOAA 10484 (N05W29) evolved on 25 October, 2003 was very complex having $\beta \gamma \delta$ configuration. This AR has produced many recurrent surges and flare activities during its passage on the solar disc. It produced major surge activities on 22 and 25 October, 2003. On October 25, we observed recurrent surges between 01:50 UT and 04:15 UT. A preliminary report on these observations has been presented in Uddin et al. (2010). In this active region, there was no evidence of strong emerging magnetic fluxes during the recurrent surges.

Many questions arise around the surge activity:

What is the trigger mechanism of the surges? Are they due to reconnection with the pre-existing field lines? What is the dynamics of the magnetic boundary bringing collision of opposite polarities? Are the pre-existing field lines open or close at the periphery of the active region? Commonly outflows are observed at the periphery of active regions (Harra et al. 2008; Del Zanna 2008). McIntosh \& De Pontieu (2009) claimed that dynamic chromospheric spicules in the outskirts of ARs are related to these outflows. Warren et al. (2011) and 
Ugarte-Urra \& Warren (2011) claimed that there is no direct relationship between these two populations of structures, i.e., the hot and cool loops maintained respectively at mega/submega-Kelvin temperatures. The questions arises that whether these observed surges in AR 10484 may participate to the commonly observed outflows in the outskirts of active regions or not? To understand this issue, statistical studies should be done to find if any relationship between these cool jets and the outflows of hot plasma commonly observed at the periphery of active regions does exist. However, this topic is out of the scope of present work.

In this paper, we present a detail multiwavelength study of recurrent surges and their associated events (e.g., flares) as occurred in AR 10484. In Section 2, we present the details of data sets used in this study. The multiwavelength evolution of the surges and their association with subflares are described in Section 3, In section 4, we describe the magnetic field evolution of active region before and during the surges and associated flares. We discuss on the possibility for a decaying active region and its magnetic activities to produce surges, and conclude, in the last section, on our results in the frame of the above questions and on the possible trigger of the surges: wave or reconnection .

\section{OBSERVATIONAL DATA SETS}

The data sets used for our present study, have been taken from following sources:

H $\alpha$ Data : The H $\alpha$ observations of the flare and associated surges were carried out at ARIES, Nainital, India by using $15 \mathrm{~cm} \mathrm{f/15} \mathrm{Coudé} \mathrm{solar} \mathrm{tower} \mathrm{telescope} \mathrm{equipped} \mathrm{with} \mathrm{H} \alpha$ filter. The image size was enlarged by a factor of two using a Barlow lens. The images were recorded by a 16 bit $576 \times 384$ pixels CCD camera system having pixel size $22 \mu^{2}$. The resolution of the images is $1^{\prime \prime}$ per pixel. The cadence for the images is $\sim 15-20$ sec.

SOHO/MDI Data : To understand the evolution of magnetic complexity of the active region, we use SOHO/MDI data. The magnetic field data was taken from the SOHO/MDI instrument (Scherrer et al.1995). The cadence of images is 96 minute and the pixel resolution is $1.98^{\prime \prime}$.

SOHO/EIT Data : SOHO/EIT (Delaboudinière et al. 1995) observe the full-disk Sun with a cadence of $12 \mathrm{~min}$ and pixel resolution of $2.5^{\prime \prime}$. It observes in four spectral bands centered

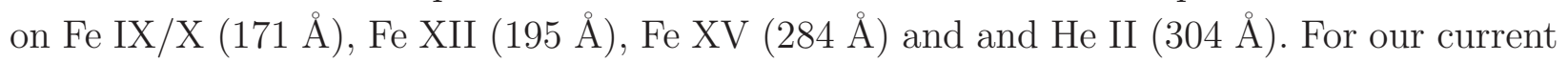
study, we used $171 \AA$ A data.

X - Ray Data : To understand the evolution of flares and associated surges, we recon- 
structed X-ray images from the Reuven Ramaty High-Energy Solar Spectroscopic Imager (RHESSI; Lin et al. (2002)). We reconstructed the images in 6-12 keV energy band from collimators ( $3 \mathrm{~F}$ to $9 \mathrm{~F}$ ) using the CLEAN algorithm, which has a spatial resolution of $\approx 7^{\prime \prime}$ (Hurford et al. 2002).

\section{MULTIWAVELENGTH OBSERVATIONS OF RECURRENT SURGES AND ASSOCIATED FLARES}

The multiwavelength evolution of the observed solar surges and their association with flares are described in following subsections.

\subsection{Temporal variations of $\mathbf{H} \alpha$ surges and flares}

The ARIES H $\alpha$ images during the surge activities on 25 October, 2003 from NOAA AR 10484, are presented in Fig. 1. They show the dynamic evolution of the recurrent surge activity from 01:50 UT to 04:15 UT. During the above mentioned time period, we observed several surges in $\mathrm{H} \alpha$. The surge activity occurred in the following satellite sunspots of the active region NOAA AR 10484.

Seven surges (Surge 1 to Surge 7) were identified (Fig. 1, see the arrows). Four of them were clearly associated with $\mathrm{H} \alpha$ brightenings. To investigate in more detail the surge evolution and the $\mathrm{H} \alpha$ brightenings at their footpoints, we computed the $\mathrm{H} \alpha$ relative intensity profile of the brightenings, the time of the $\mathrm{H} \alpha$ brightening maxima, the onsets of surges, and the time where the surge vanishes (Fig. 2 and Table 1).

In Fig. 2, we also present the GOES soft X-ray light curve obtained by full disk integration (top panel) and the RHESSI satellite thermal emission (6-12 keV) of the AR 10484 (middle panel). The latter curve has unfortunately large time gaps, however, we are able to identify two flares at $\sim 3: 00$ UT and at $\sim 4: 00$ UT. By comparing the three light curves of the Fig. 2, we conclude that all the $\mathrm{C}$ class X-ray flares of the GOES curve (Flares 1, 2, and 3 with 3 bumps) occur in AR 10484, excepted one at 02:02 UT which is observed in the neighbouring AR 10486. We present in Table 1 the class of the flares. We reconstructed images of RHESSI in the low energy band (6-12 keV) where there are enough counts.

To understand the location of the X-ray sources with $\mathrm{H} \alpha$ brightenings, we co-aligned the $\mathrm{H} \alpha$ data with the RHESSI thermal source. The contours of the X-ray sources is drawn in two $\mathrm{H} \alpha$ images shown in Fig. 3. The location of the X-ray sources coincides with the $\mathrm{H} \alpha$ brightened footpoint for the two surges corresponding to the two flares observed by RHESSI 
Table 1: Details of the surges and flares

\begin{tabular}{|c|c|c|c|c|c|c|c|c|}
\hline $\begin{array}{c}\text { Name of } \\
\text { surge }\end{array}$ & $\begin{array}{c}\text { Surge } \\
\text { onset }\end{array}$ & $\begin{array}{c}\text { H } \alpha \text { max } \\
\text { Intensity }\end{array}$ & $\begin{array}{c}\text { Max } \\
\text { GOES }\end{array}$ & $\begin{array}{c}\text { Flare } \\
\text { class }\end{array}$ & $\begin{array}{c}\text { No. of } \\
\text { Flare }\end{array}$ & $\begin{array}{c}\text { Max } \\
\text { RHESSI }\end{array}$ & $\begin{array}{c}\text { Max Surge } \\
\text { Length }\end{array}$ & Type III \\
\hline 1 & $1: 50$ & $1: 50$ & $1: 57$ & C1.2 & 1 & No & $2: 10$ & $1: 55-58$ \\
2 & $3: 05$ & $<3: 09$ & $3: 00$ & C2.6 & 2 & $3: 00$ & $3: 30$ & $3: 00$ \\
3 & $3: 36$ & $3: 30-39$ & $3: 35-3: 39$ & C3.9 & 3 (bump1) & No & $3: 40$ & $3: 35-3: 45$ \\
4 & $3: 50$ & $3: 52$ & $3: 52$ & C3.4 & 3 (bump2) & No & $4: 00$ & - \\
5 & $3: 42$ & $3: 40$ & $3: 40$ & C3.9 & 3 (bump1) & No & $4: 05$ & $3: 35-3: 45$ \\
6 & $<3: 55$ & - & - & - & - & No & $4: 09$ & - \\
7 & $4: 09$ & $4: 10$ & $3: 57$ & C3.6 & 3 (bump3) & $<4: 00$ & $4: 20$ & $4: 20$ \\
\hline
\end{tabular}

(Flare 2, and Flare 3 with three bumps). It demonstrates the co-spatiality of the thermal sources and the $\mathrm{H} \alpha$ brightenings.

\subsection{Spatial variations of $\mathrm{H} \alpha$ surges}

We describe in details each surge shown in Fig. 1 and give quantitative results in Table 1. We have calculated for each surge its extension versus time from the brightened footpoint to its leading edge and its speed. These results are shown in Fig. 2 (bottom panel) and in Table 1.

Surge 1 was a small ejection of plasma in the North-East direction, and was associated with Flare 1 and an $\mathrm{H} \alpha$ brightening (Fig. 11). Due to unavailability of high-resolution $\mathrm{H} \alpha$ observations, we could not see its evolution in details. Surge 2 started around 03:06 UT in North-East direction, increased within two periods and finally decayed around 03:30 UT. This surge was associated with Flare 2 ( C2.9) as well as with an $\mathrm{H} \alpha$ brightening at its footpoint. Surge 3 started very impulsively around 03:36 UT with a rapid enhancement of an $\mathrm{H} \alpha$ brightening (cf., Fig. 2). The plasma was ejected in North-East direction, however, slightly East in comparison to the direction of the previous surges. This surge decayed in 4 minutes around 03:40 UT. The speed of this surge expansion is estimated to $200 \mathrm{~km} / \mathrm{s}$, which is the faster. When the $\mathrm{H} \alpha$ brightening reached its maximum, the surge was decaying. It could be associated with the first bump of Flare 3 (Flare 3, bump 1). Similarly, Surge 4 started to erupt around 03:45 UT in the North-East direction from the triggering site of the recurrent surges during the impulsive and maximum phase of the $\mathrm{H} \alpha$ brightening. 
At 03:55 UT, co-temporally to Surge 4, Surges 5 and 6 have already started to erupt in the East and South-East direction. Surge 5 is bigger than Surge 6, and more elongated in size. After some time, they appeared as a single surge forming together a fan-type dark structure. Surge 5 and 6 were not associated with intense brightenings at their footpoints. However, during the surge onset period, we found faint brightenings. Possibly the flare brightening may be hidden by the dark surge material or it is associated with the first bump of Flare 3.1. Thereafter, they merge with each other, and individual surges were no longer resolved. We could only calculate the length of Surge 5 as the contrast of surge 6 was comparatively low. The length-time plot of Surge 5 shows that the surge erupt after Flare 3. Surge 7 started around 04:09 UT again in the East direction, and was initiated from a $\mathrm{H} \alpha$ brightening corresponding to Flare 3.3. Surge 7 reached the largest height compared with the other surges. The maximum projected length in case of Surge 7 is around $140 \mathrm{Mm}$ and its speed close to $100 \mathrm{~km} / \mathrm{s}$.

We conclude that the surge onsets were commonly associated with the impulsive phase of the GOES flares and reached their maximum length during the decay of $\mathrm{H} \alpha$ brightenings. Their velocities are between 25 to $200 \mathrm{~km} / \mathrm{s}$.

\subsection{SOHO/EIT observations and topology analysis}

To compare the $\mathrm{H} \alpha$ brightenings and the coronal brightenings visible in EIT, as well as surges, we overlaid EIT contours over the $\mathrm{H} \alpha$ images at a four instances. The results are shown in Fig. 4. At 03:24 UT, at 03:48 and 04:12 UT, the EIT bright regions are elongated structures and closely co-spatial with the $\mathrm{H} \alpha$ surge footpoint brightenings even they did not correspond to the onsets of the flares. The heating indicated by the brightenings in multiwavelengths is local and nearly co-spatial. The bright $\mathrm{H} \alpha$ brightenings are possibly parts of the surges, as suggested previously Schmieder et al. (1984) by looking at the Doppler shifts of such structures, which show a continuity in the Doppler-shift pattern between bright and dark structures. We would need to have informations on the Doppler shifts all along the structures to confirm that bright and dark bundles belong to a unique structure.

An interesting observation is the shape of the loops visible in EIT before the surge activity. Fig. 5 displays the SOHO/EIT $171 \AA$ image at 01:00:14 UT. The dotted ' $\mathrm{V}^{\prime}$ shaped line in the East direction indicates a fan-shape structure of magnetic field lines, which is similar to the fan-shape of the bunches of surges 4, 5, and 6. All the $\mathrm{H} \alpha$ surges erupted in the same cone direction.

A question arises: Are the field lines inside the fan open or closed? We used the Potential 
Field Source Surface (PFSS) extrapolation code to derive the global structure of the Sun on October 25, 2003. Fig. 6 shows that the bunches of field lines in the fan are in fact closed field lines linking the two active regions: AR 10484 and AR 10486.

In Fig. 4, the $\mathrm{H} \alpha$ image at 03:59 UT is overlaid by MDI contours. From this snapshot, it is shown that the footpoints of the surges are in a mixed polarity region, dominated by the positive polarity but the edge of a negative main polarity spot of the region. The region of the ejection of the surges is located between magnetic field lines on the East side of the active region and the active region itself overlaid by close loops. It means that there may be separatrix or Quasi-separatrix layers (QSLs) in that region. Field lines change easily of connectivity according to photospheric motions of the mixed polarities. It is a favourable region for reconnection. We suggest that the reconnection is done successively in different locations along the separatrix from a northern point to southern one. The material of the surge would escape along the pre-existing field lines, which make a fan-shape structure similar to the EIT loops. The reconnection would heat locally the plasma in these reconnected field lines up to $1 \mathrm{MK}$. These events are transients and may participate to the global coronal heating only with a minor effect.

\subsection{Radio Observations}

Fig. 7 (bottom-panel) shows the dynamic radio spectrum of Learmonth observatory in the frequency range 25-180 MHz. During the maximum phase of recurrent surge activities, a series of Type III bursts has been observed (Table 1). We noticed two Type III bursts during the maximum of Surge 1 before 02:00 UT. During Surge 2, two sets of Type III bursts are evident, respectively around 03:30 UT and 03:40 UT. Type III radio bursts generally represent the escaping electrons along open field lines (Dulk et al. 1979; Nitta \& De Rosa 2008). The appearance of recurrent Type III bursts during the peak evolutionary phases of the surges, may be most likely the signature of the generation of non-thermal particles due to reconnection episodes. It may be most plausible during the initiation of recurrent surge activity and the eruption of surge material that the magnetic field lines come closer and reconnect. Due to the reconnection, the energetic particles are produced and hence we observe the series of Type III radio bursts. Therefore, the presence of recurrent Type III bursts provide an evidence of multiple magnetic reconnections at the activity site, and bulk plasma acceleration along the field lines forming the surges. No Type III bursts have been identified in WIND satellite data as have shown other authors for a similar event (Bentley et al. 2000). Therefore it confirms that the accelerated particles are reaching only to the low corona, as suggested by the radio frequency range $(\sim 0.2,0.3$ solar radii). These 
particles are certainly following the system of large loops existing between the two active regions already mentioned in the previous section. These transient cool jets and associated particles are confined in close loops and cannot play an important role in the outflows observed commonly at the periphery of active regions in open field and definitively not participate to the solar wind.

\section{MAGNETIC FIELD ACTIVITY RELATED WITH RECURRENT SURGES}

In order to understand the relation between the recurrent surge activity and the magnetic field configuration of the region, we present the evolution of magnetic field before, during and after the surges (Fig. 8). From MDI time-series data, we see that a large bipole emerged ahead of a remnant active region as it crosses the limb. On the 18-20 October, the two main polarities of the emerging bipoles have a "tongue-shape" as it is frequently observed during strong emergence (Chandra et al. 2009). The polarities of the old active region broke in many segments in the surrounding of the bipole and created the $\delta$ configuration of the AR 10484. This is evident as largely extended negative polarity area surrounded by positive polarities in the active region. Already on October 22, the polarities started to disperse and the active region entered in a decay phase. Fig. 8 (top) shows the MDI magnetogram on 24 October 2003 at 14:23 UT before the surge activity. Opposite polarities surrounding the main polarities escape radially away from the spots. Around the left/right (negative/positive) main polarity, there is a ring of opposite polarities (positive/negative) respectively. Such a ring is observed successively on October 22 and on 24 October at 14:23 UT. This is the scenario of the building up of magnetic activity and complexity in and around the origin site of recurrent solar surges in AR 10484.

During the decay phase of sunspots, there is a radial dispersion of the flux with radial "sea serpent" structures with positive and negative polarities forming a ring of polarities that progressively reaches the network. This region surrounding the spot is called moat region (Harvey \& Harvey 1973). The polarities that are present in this moat region are in the dynamics of the dispersion. Looking at the MDI time-series data, we see that this ring of polarities appear several times during the dispersion phase of the magnetic fluxes. During this time, some small flux were escaping from the negative/positive main polarity and most

likely cancel with the moat polarities. This phenomena is a common process to decrease the flux during the decaying phase of an active region. The enlarged part of the active region, where the surges were originated, has been displayed in Fig. 8 (bottom).

We notice that the positive polarity P0 is submitted to the ambient dispersion process. 
P0 broke into three pieces i.e. P2, P3, and P4 on 25 October at 01:35 UT. From the main negative polarity, a small negative polarity $\mathrm{N} 1$ escaped and disappeared at 06:23 UT on 25 October. This negative polarity (N1) was being cancelled with parts of small P2 and $\mathrm{P} 3$ polarities. The cancellation of $\mathrm{P} 0$ was done in successive annihilations of positive and negative fluxes during the spatial expansion of main polarities. This annihilation occurs very close to the inversion line in close proximity of P2, P3, P4, N1, and the main negative polarity. We also followed the evolution of $\mathrm{P} 1$ and N0 polarities with time and found that these polarities are increasing in size as well in magnitude, and coming closer to each other for cancellation.

We computed the variation of magnetic fluxes in the region of these surges. For this purpose, we selected a box as shown in Fig. 8. The temporal variation of positive, negative and total fluxes before, during and after the surge activity inside the box is shown in Fig. 9 , The plot shows that the positive flux is increasing before the surge activity due to the emergence of positive ring polarities. On the other hand during the surge activity (shown by vertical line in Fig. 9), we found the decrement in positive, negative, and total flux. The constant decreasing negative flux demonstrates that the active region is in a decay phase. The decrease of total flux at the bottom of surges indicates that the flux cancellation is on going process, which would be important factor for the triggering of solar surges. However it is difficult to detect the decrease of the negative flux associated with the cancellation field at the base of the surge because the decrease of such a flux is one order of magnitude less according to the decrement of positive flux.

Under the base-line of our interpretation based on morphological investigation of MDI data as well as temporal variation of the magnetic fluxes, it may also be quite possible that the flux cancellation could be a signature of submergence of the magnetic polarities. Therefore, the energy released by reconnection in the low solar atmosphere would be transformed into thermal heating and kinetic energy. These underlying processes of surges are starting again when the ring of opposite polarities emerges around the main spots. An other important reason that such cancellation leads to surge activity, is the existence of the QSLs, region where field lines can change of connectivity.

In the present study under the baseline of our observations, we found the emergence and fragmentation of magnetic polarities, and thereafter their cancellation with the main magnetic polarities that trigger the recurrent surges and associated energy release (heating) at their footpoints. Fig. 10 displays the schematic cartoon showing the surge association due to the reconnection of the neighbouring field lines (i.e. positive) with the opposite polarity field region. The "X" symbol shows the reconnection point in between opposite polarity field regions. 


\section{DISCUSSION AND CONCLUSIONS}

In the present paper, we outline a multiwavelength study of seven recurrent surges and associated flare brightenings on October 252003 from the active region NOAA AR 10484. For this study, we use ARIES H $\alpha$, SOHO/EIT, MDI, and RHESSI observations. Out of seven surges, five surges were associated with footpoint brightenings and corresponding flare energy release. In case of surge five and six, we could not notice footpoint brightening. One possibility to not see footpoint brightening is that these surges are very dynamic and extended, as well as due to the large area of these surges the compact footpoint brightening may be hidden in the cool plasma eruption. The length-time plots of brightenings associated surges indicate that the length is increasing or sometimes decreasing (as in the case of surge 2) with the increment or decrement of the brightness at footpoint. This is the evidence that compact energy release at the base is pumping the plasma material in the form of surge. All the surges ejected in the North-East to South-East direction. The speed of the surges is between 25 and $200 \mathrm{~km} / \mathrm{s}$. This kind of large velocity has been observed in spicules Type II in Ca H as reported by De Pontieu \& McIntosh (2010). They claimed that they can follow the up flows in hot structures (1 to $2 \mathrm{MK}$ ) which could participate to the coronal heating. We cannot confirm their findings even if hot material (1MK) is observed. Recently, the hot plasma up flows were also detected near the boundary of active regions, which may be potential candidates for the coronal heating (De Pontieu et al. 2011). Such type of jets, surges, and confined plasma dynamics have been modelled recently in form of the evolution of slow shocks that could carry the hot plasma material followed by the cool plasma in the under pressure region in magnetic flux tubes (Srivastava \& Murawski 2011, 2012; Kayshap et al. 2012). Afterall, in the present observations, the hot plasma is detected at the base of the cool jets and not at the top. The shock mechanism cannot be at work in the present situation. Both hot and cool plasma follow field lines belonging to the same system joining two active regions. This plasma is not in open field lines and therefore, will not implement the commonly observed outflows in the outskirts of the active regions which could participate to the initiation of the solar wind (Harra et al. 2008; Del Zanna 2008).

The MDI magnetograms reveal positive flux of opposite polarities around the main spots. This behaviour could indicate that the active region is in a decay phase with the presence of a moat region. The polarities are very much fragmented in this moat region. The dispersion motion pushed the parasitic positive polarities to merge with negative polarities in the moat region. The positive parasitic polarity changes shape, breaking into pieces and cancel with neighbouring opposite polarities during the dispersion of the dipoles of the moat. The cancellation of flux initiates the surges. The horizontal motion of the polarities in the moat region is important to push the opposite polarities together and forces the reconnection. The field lines of the sunspot penumbra are reconnecting with the pre-existing 
open field external to the active region. It is frequently observed that surges occur at the periphery of active region close to parasitic polarities (Kurokawa et al. 2007; Brooks et al. 2007). MHD simulations show that surges and jets occur when polarities merge close to open field lines like in coronal holes (Moreno-Insertis et al. 2008). A question subsists if the surges are produced by squeezing and compression of the plasma or by reconnection (Isobe et al. 2007). Flux cancellation at the bottom of the surges might give the power for recurrent surge activities. This scenario is supported by Yokovama \& Shibata (1996) on the basis of numerical simulations. The flares that formed nearby the footpoints of the surges and the Type-III radio bursts that were observed during these events are evidences of magnetic reconnections (Shibata et al. 1994; Canfield et al. 1996b; Bentley et al. 2000). The presence of open field close to the active region favours the occurrence of surges and jets (Moreno-Insertis et al. 2008).

However, the formation of various types of jets (e.g., surges, coronal jets, spicules) at different spatio-temporal scales can either be driven by direct reconnection process, or sometimes reconnection triggered them indirectly. This depends upon the height of the reconnection site as well as the local plasma and magnetic field conditions. Sterling et al. (1993) have demonstrated that the pressure pulse generated by energy release in the nonmagnetized atmosphere can trigger the surge material. Recently, the cool jets and long spicules have been reported as driven by the reconnection generated velocity pulses sufficiently evolved in the chromosphere (Srivastava \& Murawski 2011; Murawski et al. 2011; Kayshap et al. 2012). However, such magnetohydrodynamic pulses and their steepening in form of localized shocks were associated mostly with non-flaring regions, where they attain sufficient spatio-temporal scales and ambient plasma conditions for their growth and subsequently the triggering of the various types of jets. Moreover, such types of pulse driven jets exhibit quasi-periodic rise and fall of the plasma material. In the present observations, we get the recurrent surge jets and associated flare energy release. Photospheric magnetic field cancellation due to the fragmentation of the emerging opposite polarities and associated compact flare energy release are clearly evident, which may further release the plasma along the open field lines in the form of recurrent surges. Therefore, the recurrent flare energy release at the base of the surge locations near their footpoints generated due to photospheric reconnection may be the main cause for the surge eruptions. Collision of the magnetic polarities triggers the compact flares and associated surges. Therefore, we rule out the other causes e.g., the generation of the pressure or velocity pulses in the surge region, evidence of some explosive event (Madjarska et al. 2009), role of the twist (Pariat et al. 2010) etc for the presented observations of the jet formation.

In conclusion, we report on a multiwavelength observational study of energy build-up and dynamics in the form of multiple surge eruptions associated with flares due to successive 
reconnections initiated by magnetic flux cancellations. However, the future multiwavelength observations and related MHD modeling should be carried out using recent high spatial and temporal resolution observations from space (e.g., SDO, Hinode) and complementary ground-based observations to understand the initiation, energetics, magnetic field topology and dynamics of surges.

We acknowledge the valuable suggestions of the referee that improved our manuscript considerably. The authors thank CEFIPRA Project 3704-1 for its support to this study on "Transient events in Sun Earth System" during our bilateral collaboration. We acknowledge the space borne instruments onboard SOHO, RHESSI, and ground based Learmonth, Australia for the data used in this study. SOHO is an international cooperation between ESA and NASA. BS thanks the ISSI group (Bern) lead by Klaus Galgaard on Flux emergence which helps her to have a clear idea of the development of this surge activity. AKS acknowledges Shobhna Srivastava for her patient encouragement.

\section{REFERENCES}

Beck, C., Bellot Rubio, L. R., Schlichenmaier, R., \& Sütterlin, P. 2007, A\&A, 472, 607

Bentley, R. D., Klein, K.-L., van Driel-Gesztelyi, L., Démoulin, P., Trottet, G., Tassetto, P., \& Marty, G. 2000, Sol. Phys., 193, 227

Brooks, D. H., Kurokawa, H., \& Berger, T. E. 2007, ApJ, 656, 1197

Brooks, D. H., Ugarte-Urra, I., \& Warren, H. P. 2008, ApJ, 689, L77

Canfield, R. C., Reardon, K. P., Leka, K. D., Shibata, K., Yokoyama, T., \& Shimojo, M. 1996a, ApJ, 464, 1016

Canfield, R. C., Reardon, K. P., Leka, K. D., Shibata, K., Yokoyama, T., \& Shimojo, M. 1996b, ApJ, 464, 1016

Chae, J., Qiu, J., Wang, H., \& Goode, P. R. 1999, ApJ, 513, L75

Chandra, R., Jain, R., Uddin, W., Yoshimura, K., Kosugi, T., Sakao, T., Joshi, A., \& Deshpande, M. R. 2006, Sol. Phys., 239, 239

Chandra, R., Schmieder, B., Aulanier, G., \& Malherbe, J. M. 2009, Sol. Phys., 258, 53

De Pontieu, B., \& McIntosh, S. W. 2010, ApJ, 722, 1013 
De Pontieu, B., et al. 2011, Science, 331, 55

Del Zanna, G. 2008, A\&A, 481, L49

Delaboudinière, J., et al. 1995, Sol. Phys., 162, 291

Dulk, G. A., Melrose, D. B., \& Suzuki, S. 1979, Proceedings of the Astronomical Society of Australia, 3, 375

Engell, A. J., Siarkowski, M., Gryciuk, M., Sylwester, J., Sylwester, B., Golub, L., Korreck, K., \& Cirtain, J. 2011, ApJ, 726, 12

Georgakilas, A. A., Koutchmy, S., \& Alissandrakis, C. E. 1999, A\&A, 341, 610

Harra, L. K., Sakao, T., Mandrini, C. H., Hara, H., Imada, S., Young, P. R., van DrielGesztelyi, L., \& Baker, D. 2008, ApJ, 676, L147

Harvey, K., \& Harvey, J. 1973, Sol. Phys., 28, 61

Hurford, G. J., et al. 2002, Sol. Phys., 210, 61

Isobe, H., Tripathi, D., \& Archontis, V. 2007, ApJ, 657, L53

Kayshap, P., Srivastava, A. K., \& Murawski, K. 2012, ApJ, submitted

Kitiashvili, I. N., Bellot Rubio, L. R., Kosovichev, A. G., Mansour, N. N., Sainz Dalda, A., \& Wray, A. A. 2010, ApJ, 716, L181

Kubo, M., Lites, B. W., Shimizu, T., \& Ichimoto, K. 2008, ApJ, 686, 1447

Kurokawa, H., \& Kawai, G. 1993, in Astronomical Society of the Pacific Conference Series, Vol. 46, IAU Colloq. 141: The Magnetic and Velocity Fields of Solar Active Regions, ed. H. Zirin, G. Ai, \& H. Wang, 507

Kurokawa, H., Liu, Y., Sano, S., \& Ishii, T. T. 2007, in Astronomical Society of the Pacific Conference Series, Vol. 369, New Solar Physics with Solar-B Mission, ed. K. Shibata, S. Nagata, \& T. Sakurai, 347

Lin, R. P., et al. 2002, Sol. Phys., 210, 3

Liu, Y., \& Kurokawa, H. 2004, ApJ, 610, 1136

Madjarska, M. S., Doyle, J. G., \& de Pontieu, B. 2009, ApJ, 701, 253

McIntosh, S. W., \& De Pontieu, B. 2009, ApJ, 706, L80 
Moreno-Insertis, F., Galsgaard, K., \& Ugarte-Urra, I. 2008, ApJ, 673, L211

Murawski, K., Srivastava, A. K., \& Zaqarashvili, T. V. 2011, A\&A, 535, A58

Nitta, N. V., \& De Rosa, M. L. 2008, ApJ, 673, L207

Pariat, E., Antiochos, S. K., \& DeVore, C. R. 2010, ApJ, 714, 1762

Rachmeler, L. A., Pariat, E., DeForest, C. E., Antiochos, S., \& Török, T. 2010, ApJ, 715, 1556

Roy, J. R. 1973, Sol. Phys., 28, 95

Rust, D. M. 1968, in IAU Symposium, Vol. 35, Structure and Development of Solar Active Regions, ed. K. O. Kiepenheuer, 77

Sainz Dalda, A., \& López Ariste, A. 2007, A\&A, 469, 721

Scherrer, P. H., et al. 1995, Sol. Phys., 162, 129

Schmieder, B., Golub, L., \& Antiochos, S. K. 1994, ApJ, 425, 326

Schmieder, B., Mein, P., Martres, M. J., \& Tandberg-Hanssen, E. 1984, Sol. Phys., 94, 133

Schmieder, B., Mein, P., Simnett, G. M., \& Tandberg-Hanssen, E. 1988, A\&A, 201, 327

Schmieder, B., Shibata, K., van Driel-Gesztelyi, L., \& Freeland, S. 1995, Sol. Phys., 156, 245

Shibata, K., et al. 1992, PASJ, 44, L173

Shibata, K., Nishikawa, T., Kitai, R., \& Suematsu, Y. 1982, Sol. Phys., 77, 121

Shibata, K., Nitta, N., Strong, K. T., Matsumoto, R., Yokoyama, T., Hirayama, T., Hudson, H., \& Ogawara, Y. 1994, APJL, 431, L51

Srivastava, A. K., \& Murawski, K. 2011, A\&A, 534, A62

Srivastava, A. K., \& Murawski, K. 2012, ApJ, 744, 173

Sterling, A. C. 2000, Sol. Phys., 196, 79

Sterling, A. C., Shibata, K., \& Mariska, J. T. 1993, ApJ, 407, 778

Uddin, W., Jain, R., Yoshimura, K., Chandra, R., Sakao, T., Kosugi, T., Joshi, A., \& Despande, M. R. 2004, Sol. Phys., 225, 325 
Uddin, W., Kumar, P., Srivastava, A. K., \& Chandra, R. 2010, in Magnetic Coupling between the Interior and Atmosphere of the Sun, ed. S. S. Hasan \& R. J. Rutten, 478

Ugarte-Urra, I., \& Warren, H. P. 2011, ApJ, 730, 37

Warren, H. P., Ugarte-Urra, I., Young, P. R., \& Stenborg, G. 2011, ApJ, 727, 58

Yokoyama, T., \& Shibata, K. 1996, Astrophysical Letters Communications, 34, 133

Yoshimura, K., Kurokawa, H., Shimojo, M., \& Shine, R. 2003, PASJ, 55, 313 


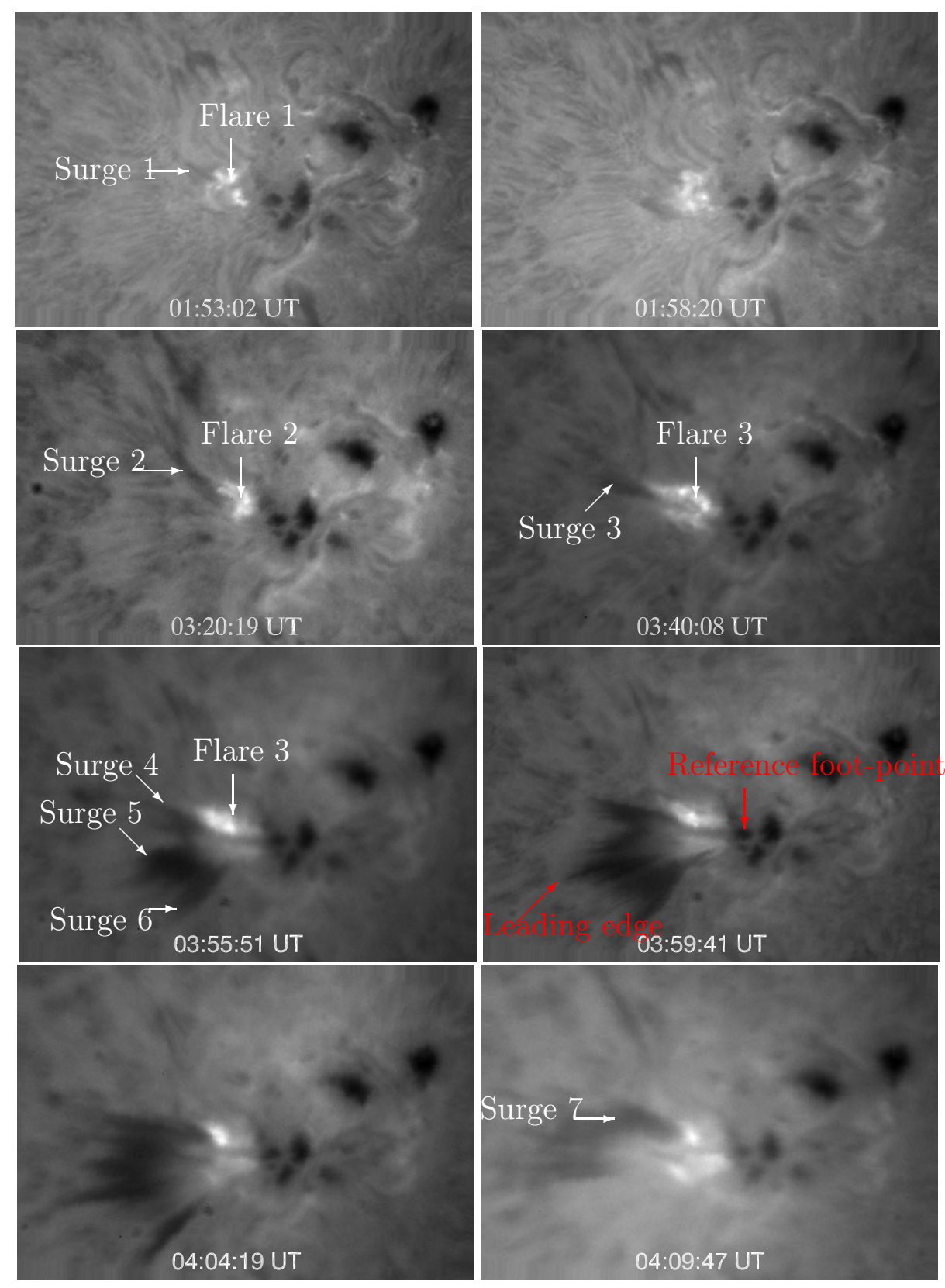

Fig. 1.- $\mathrm{H} \alpha$ image sequence showing the recurrent flare/surge activities on 25 October, 2003 in AR 10484. The field-of-view of each image is $320^{\prime \prime} \times 200^{\prime \prime}$. 


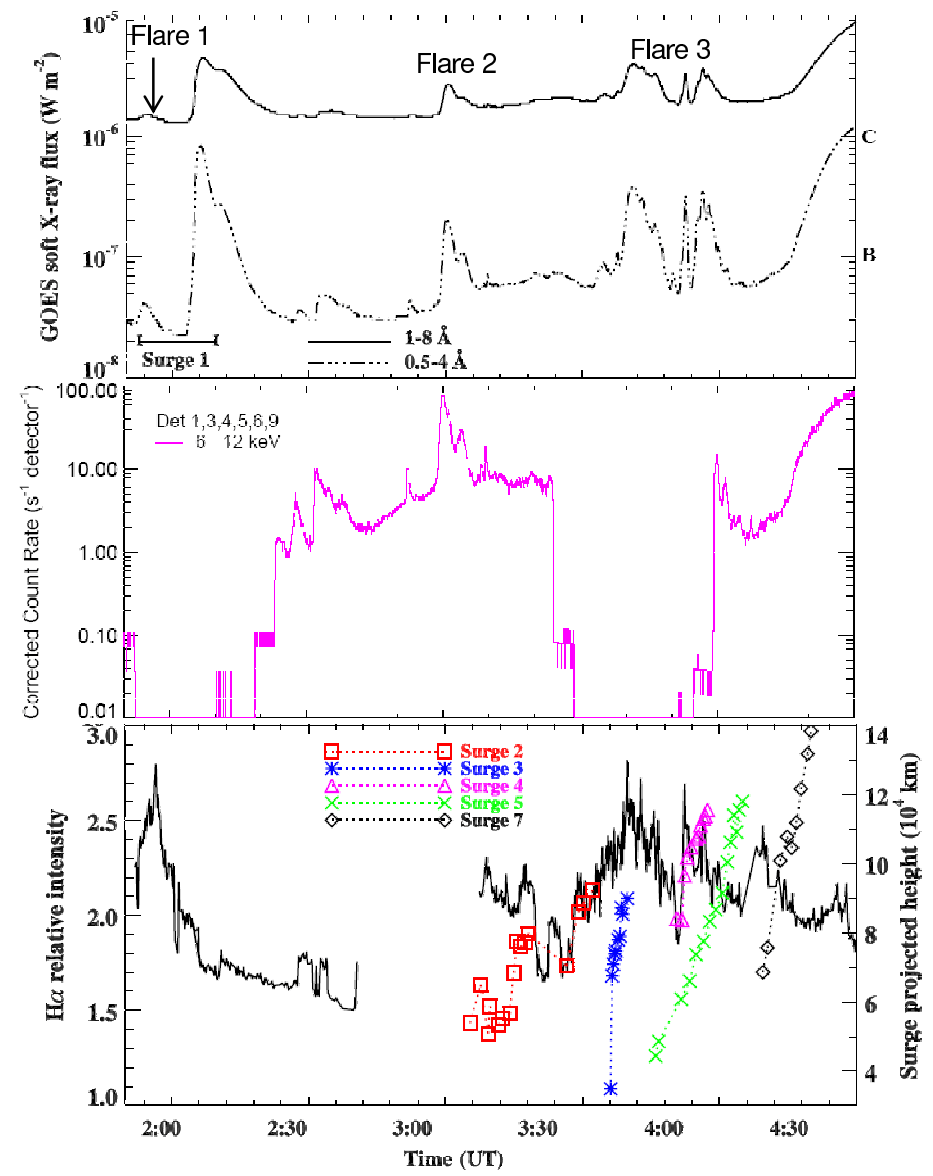

Fig. 2.- GOES Soft X-ray flux (top panel) profiles in two different wavelengths on 25 October 2003. The $\mathrm{H} \alpha$ relative intensity profile is well correlated with the GOES flux (bottom panel). There was $\mathrm{H} \alpha$ data gap in between 02:40-03:05 UT. There was recurrent small surge activities associated with flares at 01:55 and between 03:00 UT and 04:30 UT (Flares 1, 2, 3). The C4.3 flare during 02:02-02:12 was occurred in another AR NOAA 10486 located at the eastern limb. The length-time plot of the surges is shown and demonstrate the surge-flare relationship. 

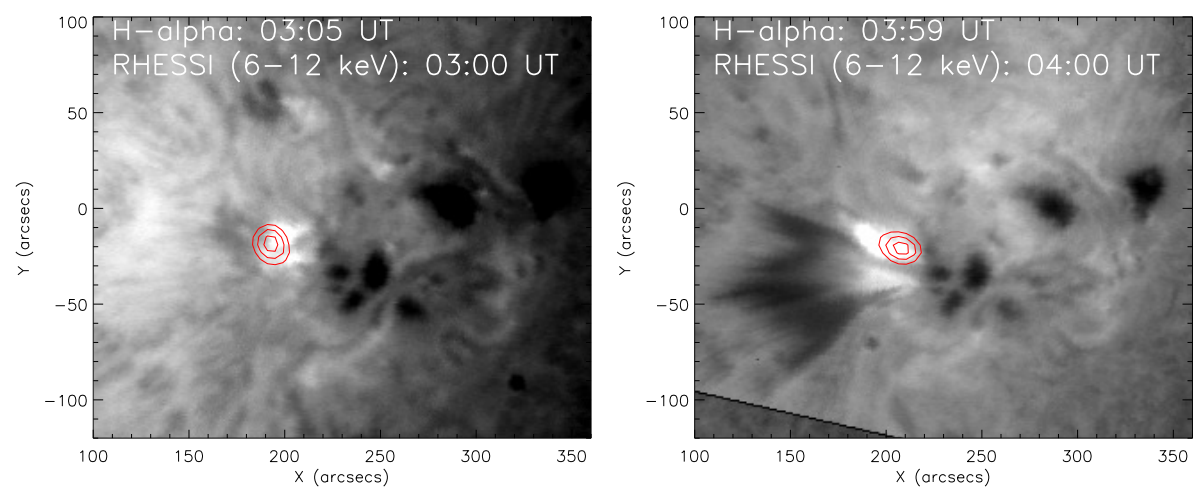

Fig. 3.- $\mathrm{H} \alpha$ images overlaid by RHESSI 6-12 keV contours during Flare 2 and Flare 3 (bump 3)The contour levels are 50, 70 and 90\% of the peak intensity.
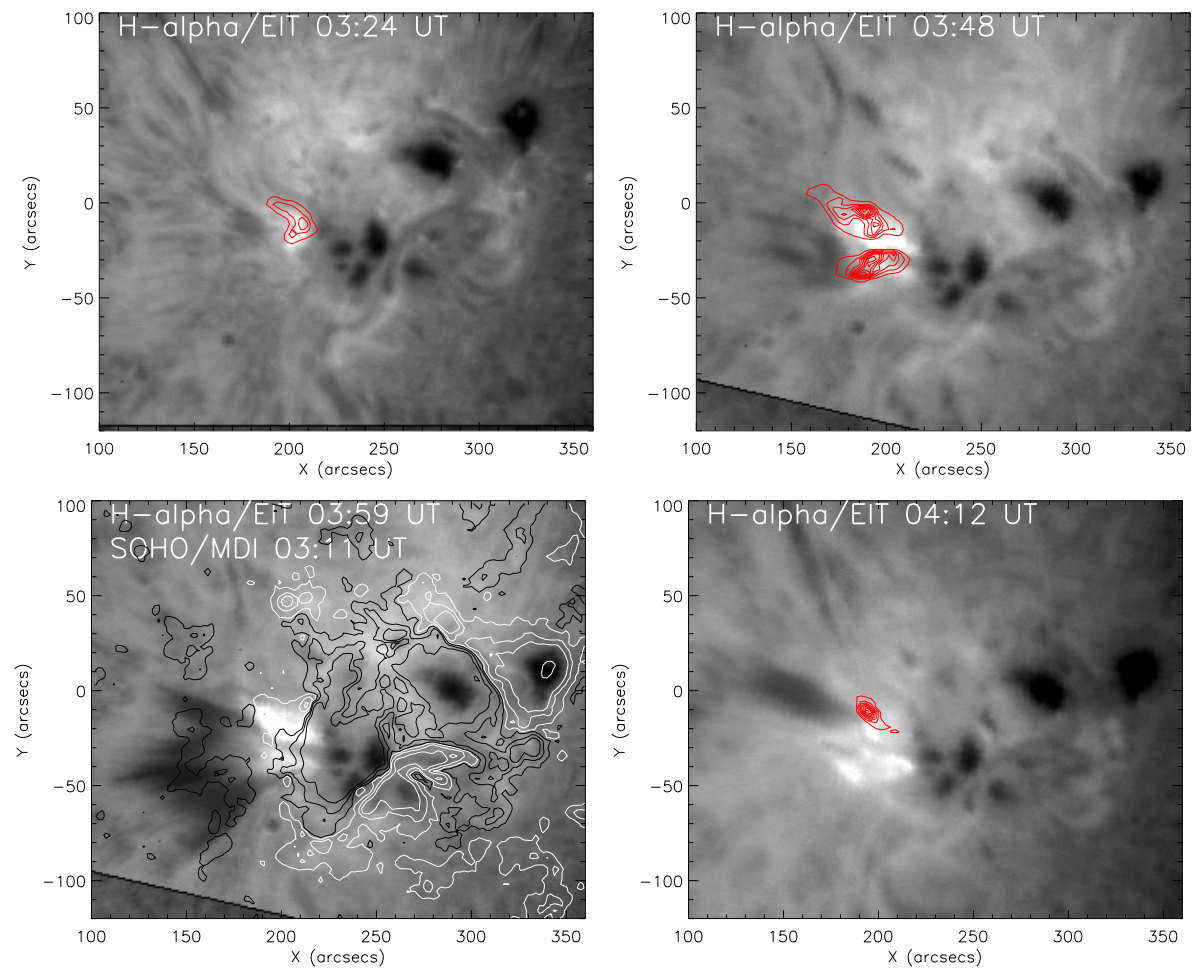

Fig. 4.- $\mathrm{H} \alpha$ images showing the flares and surge eruptions overlaid by the SOHO/EIT 171 $\AA$ contours for three different times (top, and bottom right panel). The bottom left image shows an $\mathrm{H} \alpha$ image overlaid by MDI contours (white contours show the positive polarity whereas black contours indicate the negative polarity). 


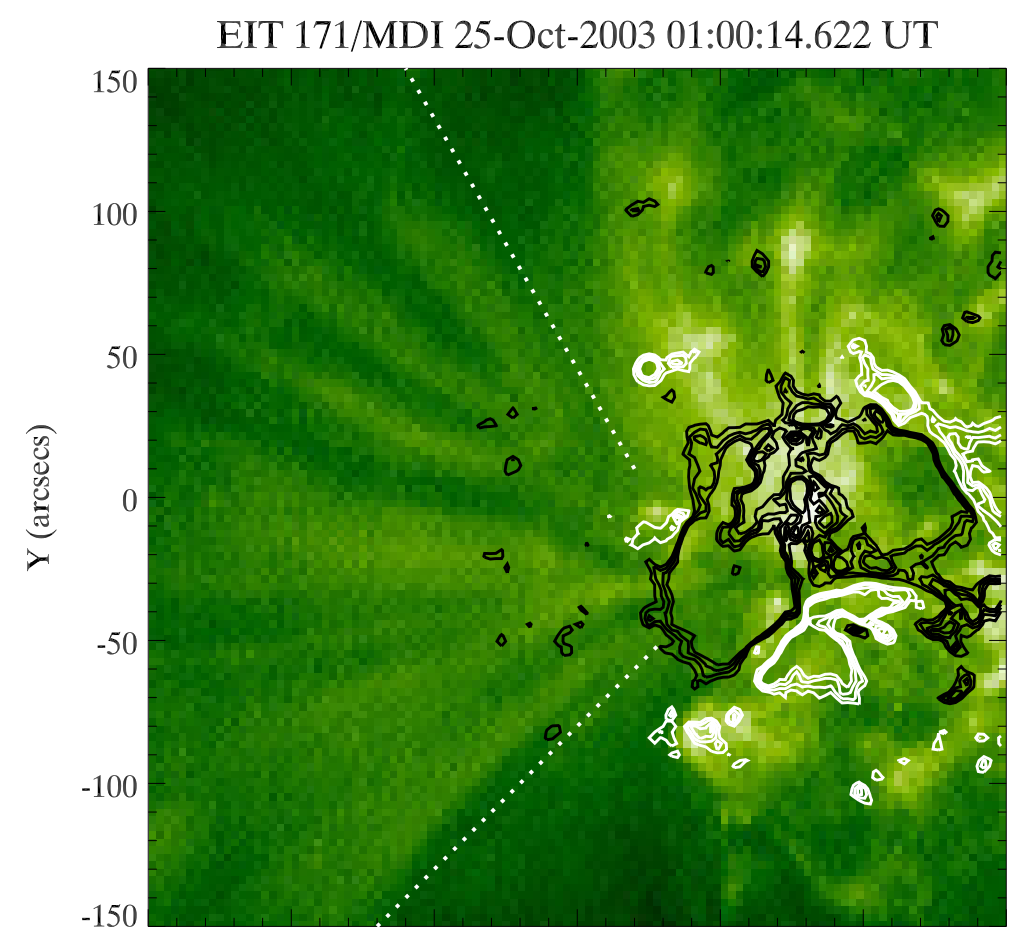

Fig. 5.- SOHO/EIT $171 \AA$ image overlaid by the MDI contours (white shows the positive polarity whereas black indicates the negative polarity). The dotted lines indicate the fanshaped structure of field lines over the activity site before the flare and surge activities. 


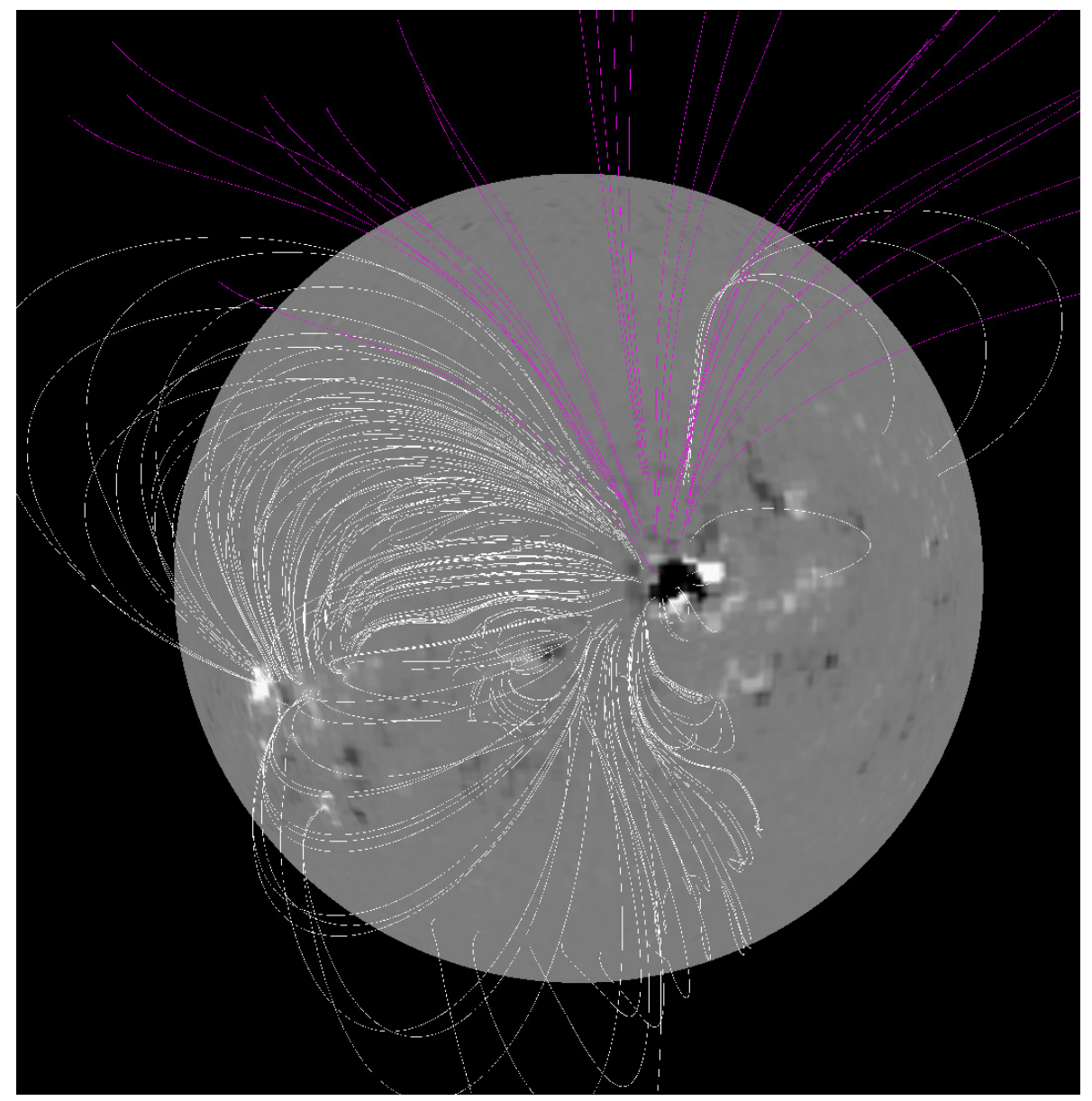

Fig. 6.- Potential Field Source Surface (PFSS) extrapolation overplotted at the SoHO/MDI full disk map on October 252003 on 00:04 UT, which shows the large-scale field connectivity of two active regions as well as the topology of magnetic field near the surge productivity site in AR10484. 


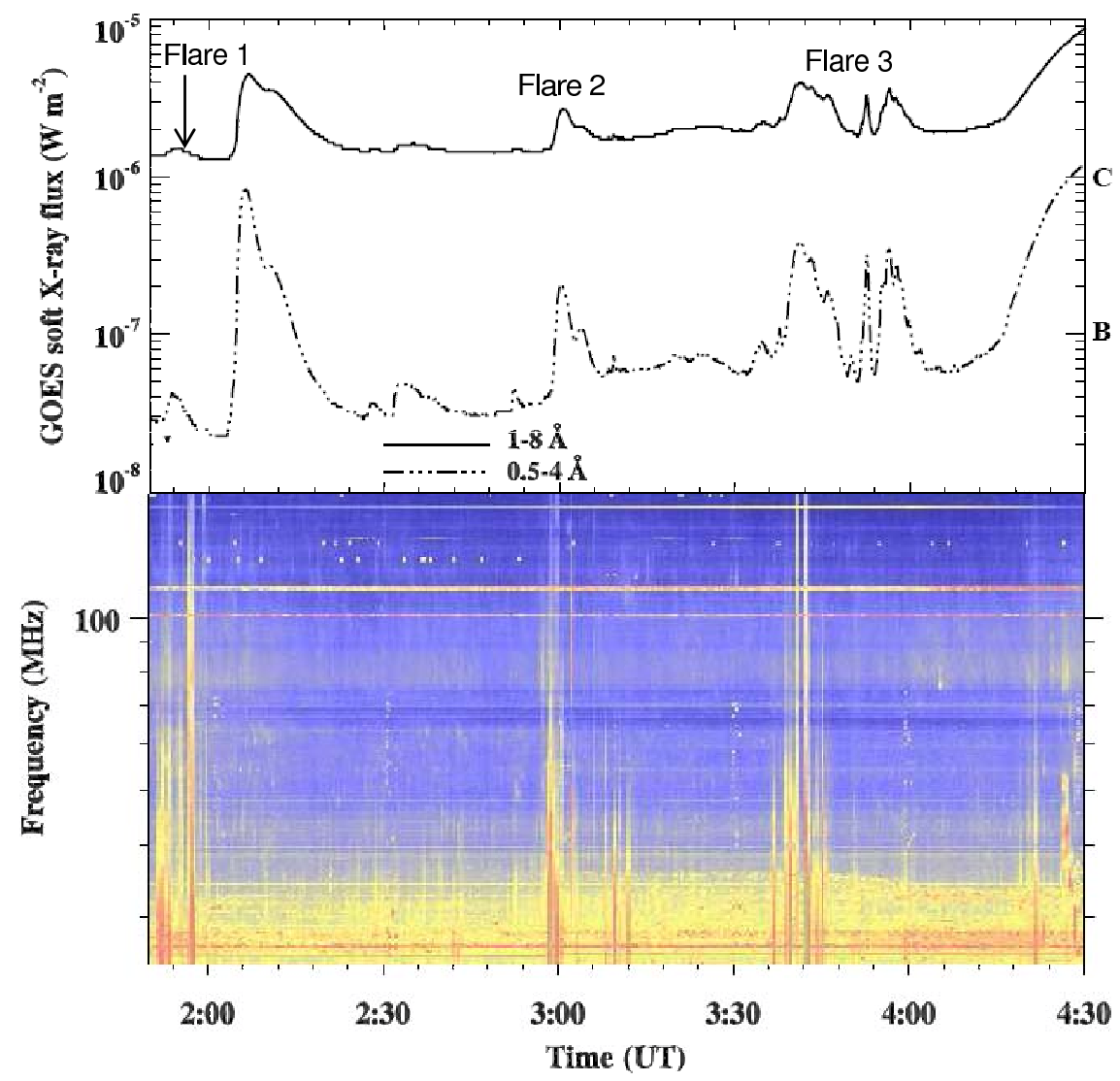

Fig. 7.- GOES soft X-ray light curve and Radio TYPE III bursts ( Learmonth) 

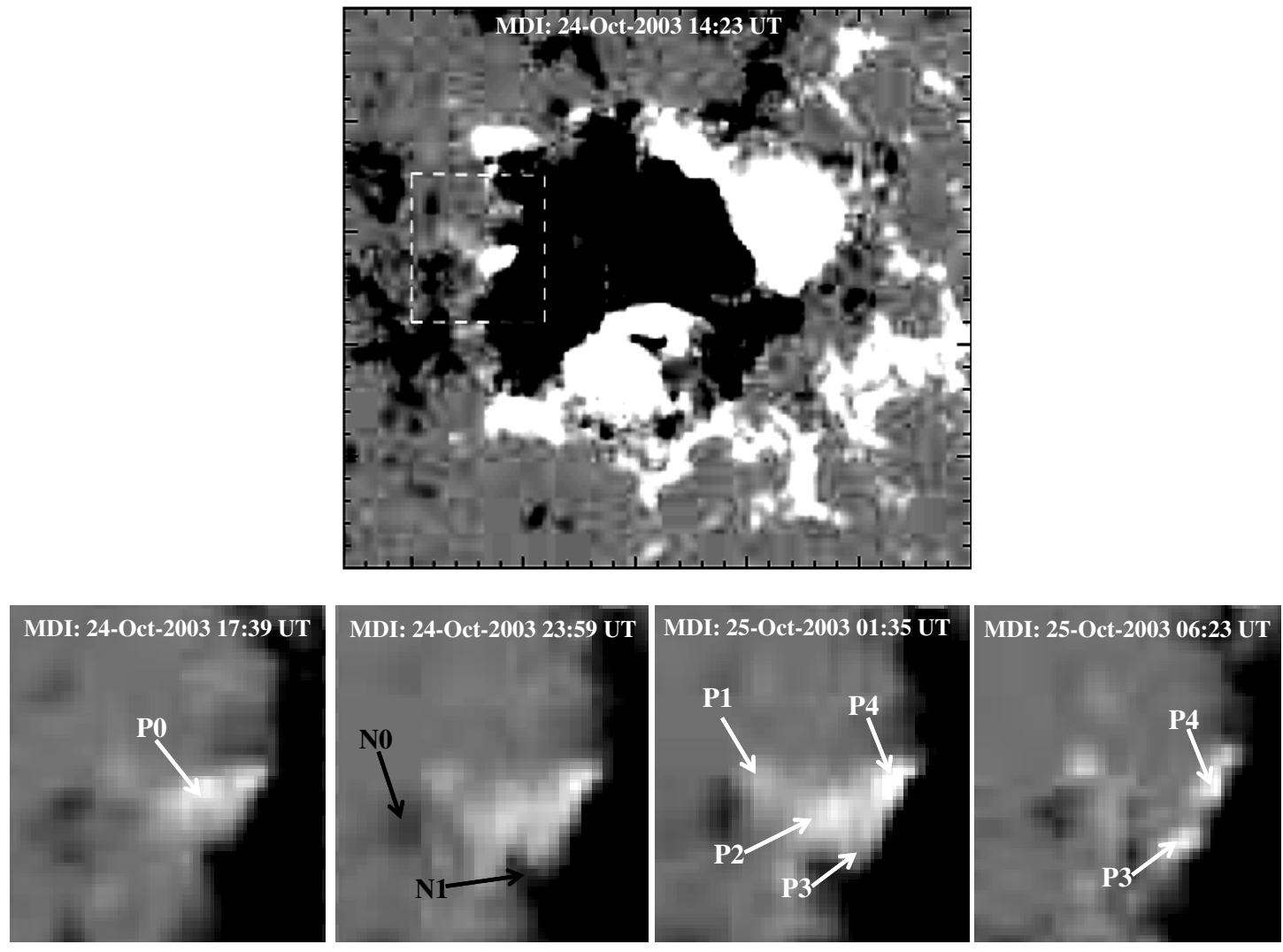

Fig. 8.- Magnetogram on 24 October, 2003 before the surge activity showing the moat region around the main polarities (top panel) and zoom on the region of the surges (presented as a white box in the top panel) before, during, and after the surges (bottom panel). The field-of-view of the upper and lower images is $280^{\prime \prime} \times 250^{\prime \prime}$ and $60^{\prime \prime} \times 65^{\prime \prime}$ respectively. 


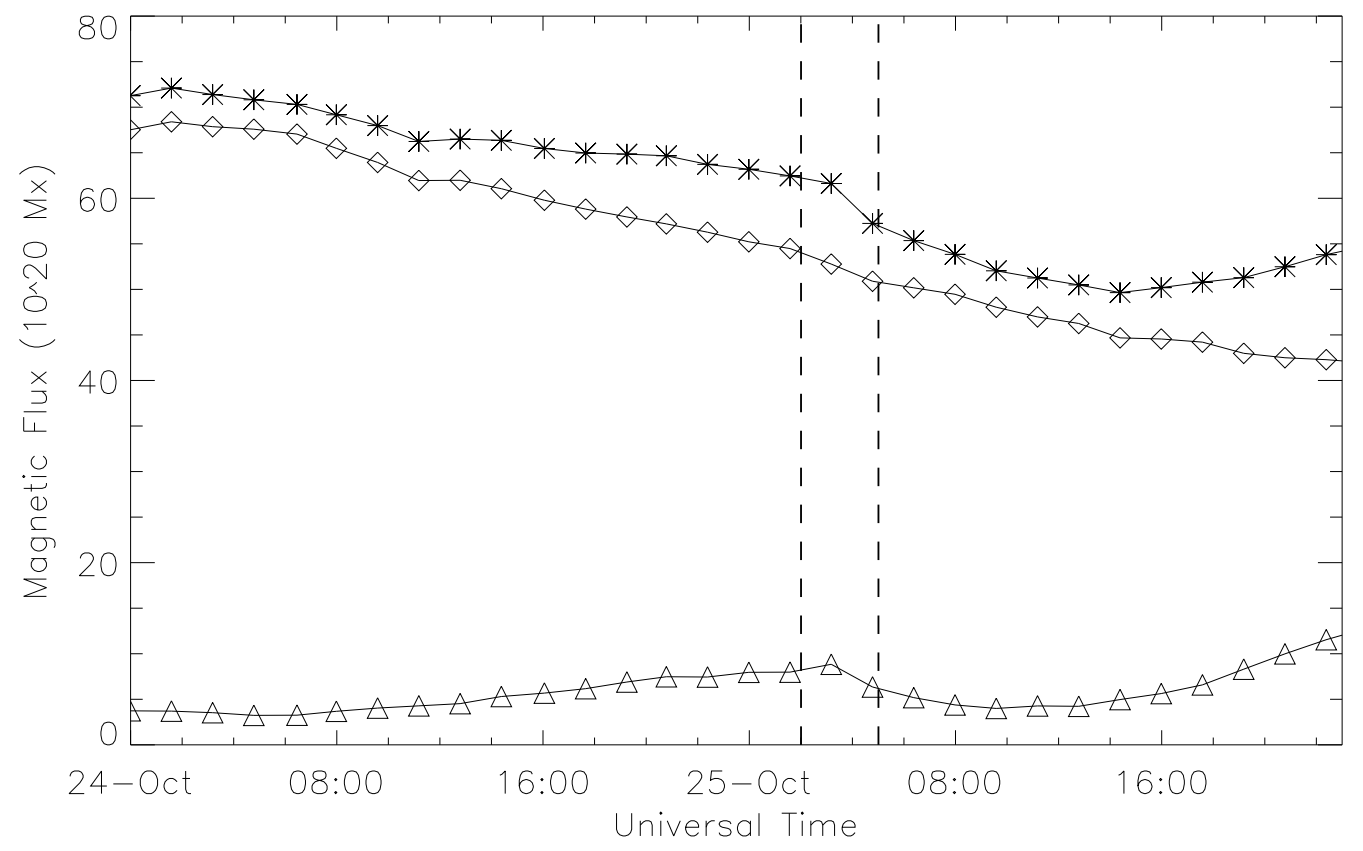

Fig. 9. - The temporal variation of positive (triangle), negative (diamond) and total (star) magnetic flux before, during, and after the surge activities as measured over the selected area shown in the bottom panels of Figure 8. The two vertical lines indicate the duration of surge activity. 


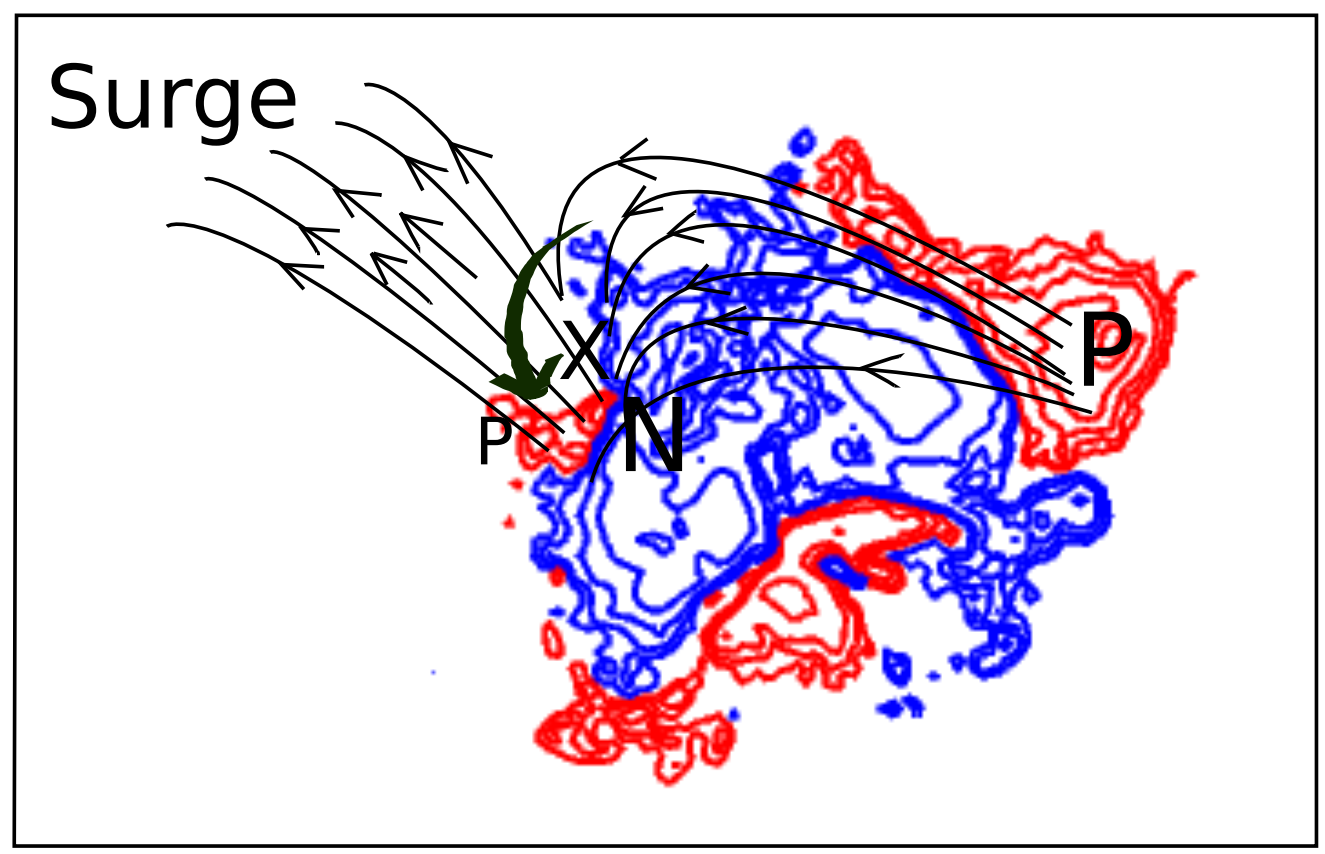

Fig. 10. - Schematic cartoon showing the surge initiation due to the reconnection (shown by ' $\mathrm{X}^{\prime}$ ) of the field lines with the opposite polarity field region, resulting of the anticlockwise motion of the negative polarity field region (indicated by thick arrow). Red contours indicate the positive polarity whereas blue ones show the negative polarity field region. Surge moves along the open field lines. 